\title{
Association between 17q25.3-rs6465657 polymorphism and prostate cancer susceptibility: a meta-analysis based on 19 studies
}

This article was published in the following Dove Press journal:

Onco Targets and Therapy

22 July 2016

Number of times this article has been viewed

\author{
Xiao Jiang ${ }^{1,2}$ \\ Mei Zhang ${ }^{3}$ \\ Xiao-Yan Bai' \\ Shujing $\mathrm{Li}^{\prime}$ \\ Huijian $\mathrm{Wu}^{\prime}$
}

'Laboratory of Molecular Medicine \& Pharmacy, School of Life Science and Biotechnology, Dalian University of Technology, Dalian, People's Republic of China; ${ }^{2}$ Department of Gastroenterology, Dalian Municipal Central Hospital Affiliated of Dalian Medical University, Dalian, People's Republic of China; ${ }^{3}$ Department of Biochemistry and Molecular Biology, Heilongjiang University of Chinese Medicine, Haerbin, People's Republic of China
Correspondence: Huijian Wu; Shujing Li School of Life Science and Biotechnology, Dalian University of Technology,

Number 2, Ling Gong Road, Dalian

I I 6024, People's Republic of China

Tel +86 4II 84706105

Fax +86 4II 84706105

Email wuhj@dlut.edu.cn; Isj@dlut.edu.cn
Background: Genome-wide association studies have identified rs6465657 polymorphism at chromosome 17q25.3 as a new prostate cancer (PCa) susceptibility locus in people of European descent. However, subsequent replication studies have yielded inconsistent results among different ethnicities. In this study, a comprehensive meta-analysis was conducted to systematically evaluate the relationship between rs6465657 polymorphism and PCa risk.

Methods: All the articles involved were identified from PubMed, EMBASE, Web of Science, EBSCO databases, and Google Scholar before December 2015. The odds ratios (ORs) with corresponding $95 \%$ confidence internals (95\% CIs) were pooled under the allele model. Fourteen eligible articles with 19 studies were finally included.

Results: In the overall population, the 17q25.3-rs6465657C allele was found to be significantly associated with increased risk of $\mathrm{PCa}$ compared to the T allele (OR $=1.097 ; 95 \% \mathrm{CI}$ : 1.061-1.134; $P<0.001)$. In the subgroup analysis stratified by ethnicity, significantly increased risk was found in the Caucasian population ( $\mathrm{OR}=1.120 ; 95 \% \mathrm{CI}$ : $1.078-1.162 ; P<0.001$ ), while the difference of OR did not reach the statistical significance in the Asian or African-American population. The analyses of sensitivity indicated the robust stability of the results, and the Begg's and Egger's test indicated that no publication bias existed.

Conclusion: The current meta-analysis suggested that the 17q25.3-rs6465657 polymorphism could be associated with PCa susceptibility, especially in the Caucasians, while this association might be different in other ethnicities.

Keywords: rs6465657, LMTK2, polymorphism, prostate cancer, risk, meta-analysis

\section{Introduction}

Prostate cancer (PCa) is the most common solid neoplasm among men in Western countries and is the sixth leading cause of cancer death in males around the world. It was reported that more than 14 million new cases of PCa and approximately 8.2 million cancer deaths due to PCa occurred in 2012 worldwide. ${ }^{1,2}$ The pathogenesis of PCa has not been elucidated yet, but it is generally considered to be caused by both environmental and genetic factors. ${ }^{3}$ Family history is acknowledged to be one of the strong risk factors, and about a twofold increased risk for $\mathrm{PCa}$ incidence is observed among patients who have a first-degree relative with $\mathrm{PCa} .{ }^{4}$ Lichtenstein et $\mathrm{al}^{5}$ reported that approximately $42 \%$ of variation underlying prostate cancer liability in a Nordic twin cohort could be explained by genetic factors. Despite this, family and twin studies have not identified reproducible susceptibility loci associated with PCa risk, suggesting that predisposition was likely mediated through a combination of multiple, common low-penetrance genetic variants. ${ }^{6}$ 
To identify the common genetic variants of $\mathrm{PCa}$, largescale genome-wide association studies (GWAS) have been conducted that reported a series of common PCa susceptibility genes in the Caucasian population, such as THADA, ITGA6, MDM4, MSMB, LMTK2, and KLK3, 4,7,8 Among these, the expression level of LMTK2 (lemur tyrosine kinase 2L) mRNA in PCa tissue was found to be significantly lower than that in nonmalignant tissues. It has been suggested that the downregulation of LMTK2 might contribute to PCa formation. ${ }^{9}$ A single-nucleotide polymorphism (SNP) rs6465657 T $>\mathrm{C}$ in the intron 9 of $L M T K 2$ at chromosome 7q21.3, which was initially identified by Eeles et al ${ }^{7}$ in a GWAS study, was reported to be associated with PCa risk. Although several subsequent replication studies have been performed to confirm this relationship, the results remain controversial. Lindstrom et al ${ }^{10}$ and Lange et $\mathrm{al}^{11}$ identified strong evidence of the association between rs6465657C and PCa risk in the US population, and the study performed by Kote-Jarai et $\mathrm{al}^{12}$ in a multiethnic population supported this conclusion; however, the other studies reported weak or no statistically significant association between 17q25.3-rs6465657C and PCa susceptibility.,13-22

To our knowledge, only one meta-analysis studied the relationship between rs6465657 polymorphism and $\mathrm{PCa}$ risk, and this showed no evidence of this association as it was restricted by a relatively small sample size. ${ }^{23}$ In addition, cumulative meta-analysis, Egger's regression, and sensitivity analyses were not carried out in the previous meta-analysis. Owing to the fact that more studies have been conducted to verify the susceptibility of rs6465657C to PCa among different ethnic populations recently, there is a need to derive a more precise evaluation of this relationship. Therefore, we performed an updated comprehensive meta-analysis to reassess the association between the 7q21.3-rs6465657 polymorphism and PCa susceptibility.

\section{Materials and methods}

\section{Literature search strategy}

To obtain all relevant articles that had investigated the association between the polymorphism of rs6465657 and PCa risk, we carried out a systematic search of publications using PubMed, EMBASE, EBSCO, and Web of Science databases and Google Scholar before April 2015, without language restriction. The search query combined the following terms of "17q25.3 or 17q25 or rs6465657 or LMTK2" and "prostate cancer* or PCa or prostatic cancer or prostatic carcinoma". The references listed from the reviews and relevant studies were also scrutinized to find additional articles related to the topic under consideration. Two investigators retrieved studies in duplicate independently.

\section{Inclusion and exclusion criteria}

The inclusion criteria adopted for the current meta-analysis were as follows: 1) studies evaluating the association between the SNP rs6465657 T $>$ C and PCa risk; 2) casecontrol or cohort studies; 3) humans studies; 4) studies with PCa diagnosis criteria based on histopathology; and 5) studies providing sufficient published data to calculate odds ratios (ORs) and the corresponding 95\% confidence intervals (95\% CIs).

The major reasons for exclusion were the following: 1) case-only studies; 2) family-based studies; 3 ) reviews, comments, meeting abstracts, meta-analyses; 4) duplicate studies, if more than one article with the same data was published, the study with the full-scale data was adopted in this meta-analysis; 5) studies of relationships between other susceptibility loci and $\mathrm{PCa}$ incidence; and 6) deviation from Hardy-Weinberg equilibrium in controls.

\section{Assessment of study quality}

The Newcastle-Ottawa scale (NOS) was adopted to evaluate the quality of the involved articles, which contained three criteria: selection, comparability, and exposure. The scores of NOS range from zero to nine. Studies with a score $\geq 5$ are considered to be high quality. ${ }^{24}$ In case of any discrepancies, an agreement was reached by discussion among all investigators.

\section{Data extraction}

Two investigators (Xiao Jiang and Xiao-Yan Bai) reviewed and extracted data from all the eligible articles independently. For each study, the variables were gathered as follows: the first author, year of publication, country, ethnicity of the participants, genotyping method, source of controls, age, and number of cases and controls. If one article contained two or more case-control groups, they were considered as two or more independent studies.

\section{Statistical analysis methods}

The frequency of 17q25.3-rs6465657C allele was calculated in the control group of each study. ${ }^{25}$ The prevalence rates of the $\mathrm{C}$ allele obtained from the individual studies were pooled together in the overall population or in various ethnic populations using the random-effects model when heterogeneity existed.

The association between rs6465657 polymorphism and susceptibility to PCa was assessed by OR and the corresponding $95 \%$ CI for each study. The significance of the pooled OR was examined by the $Z$-test. A $P$-value less than 0.05 was considered to be statistically significant. We also performed the subgroup analysis according to ethnicity. 
Heterogeneity of the eligible studies was evaluated by the Cochran's $Q$ statistical test, with significance set at the level of $P<0.1 .^{26}$ The $I^{2}$ index, expressed as a percentage, quantified the degree of heterogeneity: $0 \%-25 \%$, no heterogeneity; $25 \%-50 \%$, moderate heterogeneity; $50 \%-75 \%$, large heterogeneity; and $75 \%-100 \%$, extremely large heterogeneity. When the heterogeneity was negligible, the fixed-effects model was employed to pool the ORs of the involved studies (Mantel-Haenszel method), ${ }^{27}$ otherwise, the random-effects model (DerSimonian-Laird method) ${ }^{28}$ was used. In case of heterogeneity, meta-regression analysis was performed to determine the source of heterogeneity. The year of publication, the ethnicity (Caucasian, Asian, or African-American), source of controls, genotyping method, and sample size (defined as sample size less than 500 cases, or more than 500 cases) were chosen as common variables which might be correlated to heterogeneity, ${ }^{25,29}$ assessed by meta regression, and $P<0.05$ was considered to be statistical difference.

Sensitivity analysis was conducted to evaluate the stability of the results by removing each individual study in turn and recalculating the ORs and corresponding 95\% CIs. ${ }^{30}$ Cumulative meta-analysis was then performed to estimate the trend of the pooled OR for rs6465657 polymorphism and PCa susceptibility sorted by sample size.

The Begg's funnel plot $^{31}$ and the Egger's regression ${ }^{32}$ were applied to evaluate publication bias among the involved articles, with $P<0.05$ being considered as significant publication bias. STATA software, version 12.0 (Stata Corporation, College Station, TX, USA) was used to conduct all the statistical analyses.

\section{Results}

\section{Characteristics of the included studies}

A total of 136 articles were originally obtained from PubMed, EMBASE, EBSCO, Web of Science databases, and Google Scholar based on the keyword search, and two articles were also obtained from review articles. ${ }^{33,34}$ Eighty-three articles were left after removing the duplicates. After excluding reviews, comments, meta-analyses, and meeting abstracts, as well as the obviously irrelevant articles by scanning the titles and abstracts, 29 articles were retained, which potentially studied the association between rs6465657 polymorphism and PCa risk. However, nine articles that did not study this exact topic after reading the full text of the articles, three studies which did not provide ORs and corresponding $95 \%$ CIs, ${ }^{33,35,36}$ together with two family-based studies ${ }^{37,38}$ were also excluded. Finally, 14 eligible articles, $, 10-21,22$ providing 19 case-control studies with 41,755 patients and
78,967 controls were included in the current meta-analysis. The systematic search and selection process for the association between rs6465657C and susceptibility to $\mathrm{PCa}$ is displayed in Figure 1.

The sample size of the included studies varied from 221 to 37,082. The related ethnicities of the study subjects were Caucasian ( $n=9)$, Asian ( $n=5)$, African-American $(n=2)$, and multiethnicity $(n=3)$. All the articles involved in the current meta-analysis were of high quality owing to the fact that the score of each article based on NOS assessment was higher than 5 points. The main characteristics of the included studies are summarized in Table 1, and the scores of NOS among the eligible articles are listed in Table 2.

\section{Frequency of I7q25.3-rs6465657C allele in the controls}

The $\mathrm{C}$ allele of rs6465657 was chosen as the putative risk allele, and its frequency varied widely in the controls among different ethnicities. In the overall control population, the frequency of $\mathrm{C}$ allele was $63.3 \%$ (95\% CI: $56.1 \%-70.4 \%$ ) with the random-effects model, and heterogeneity was obviously present $\left(P<0.001, I^{2}=99.7 \%\right)$. For Caucasian controls, the frequency of $\mathrm{C}$ allele was $45.5 \%$ (95\% CI: $43.7 \%-47.2 \%$ ), which was lower than that in Asian controls (87.9\%; 95\% CI: 85.9\%-89.9\%) and African-American controls $(85.4 \%$; 95\% CI: 83.3\%-87.7\%). The heterogeneity was significant in Caucasian $\left(P<0.001, P^{2}=93.0 \%\right)$ and Asian controls $\left(P<0.05, P^{2}=62.1 \%\right)$, whereas no heterogeneity was detected in African-American controls $\left(P>0.05, I^{2}=0.0 \%\right.$; Figure 2).

\section{Association between 17q25.3-rs6465657 polymorphism and $\mathrm{PCa}$ risk}

The $\mathrm{C}$ variant of 17q25.3-rs6465657 was found to be a risk factor for PCa susceptibility compared to the $\mathrm{T}$ allele under the allele model according to the overall $\mathrm{OR}(\mathrm{OR}=1.097$; 95\% CI: $1.061-1.134 ; P=0.000$ ) with the random-effects model due to significant heterogeneity among studies. Furthermore, in the subgroup analysis stratified by ethnicity, significant association between rs6465657 polymorphism and PCa risk was observed restricted to Caucasian population (OR $=1.120 ; 95 \%$ CI: $1.078-1.162 ; P=0.000)$, while no associations were found in Asian and African-American populations ( $P>0.05$; Figure 3$)$. The main results of this pooled analysis are listed in Table 3.

\section{Heterogeneity and sensitivity analyses}

As shown previously, the rs6465657 polymorphism significantly associated with the increased risk of $\mathrm{PCa}$ in 


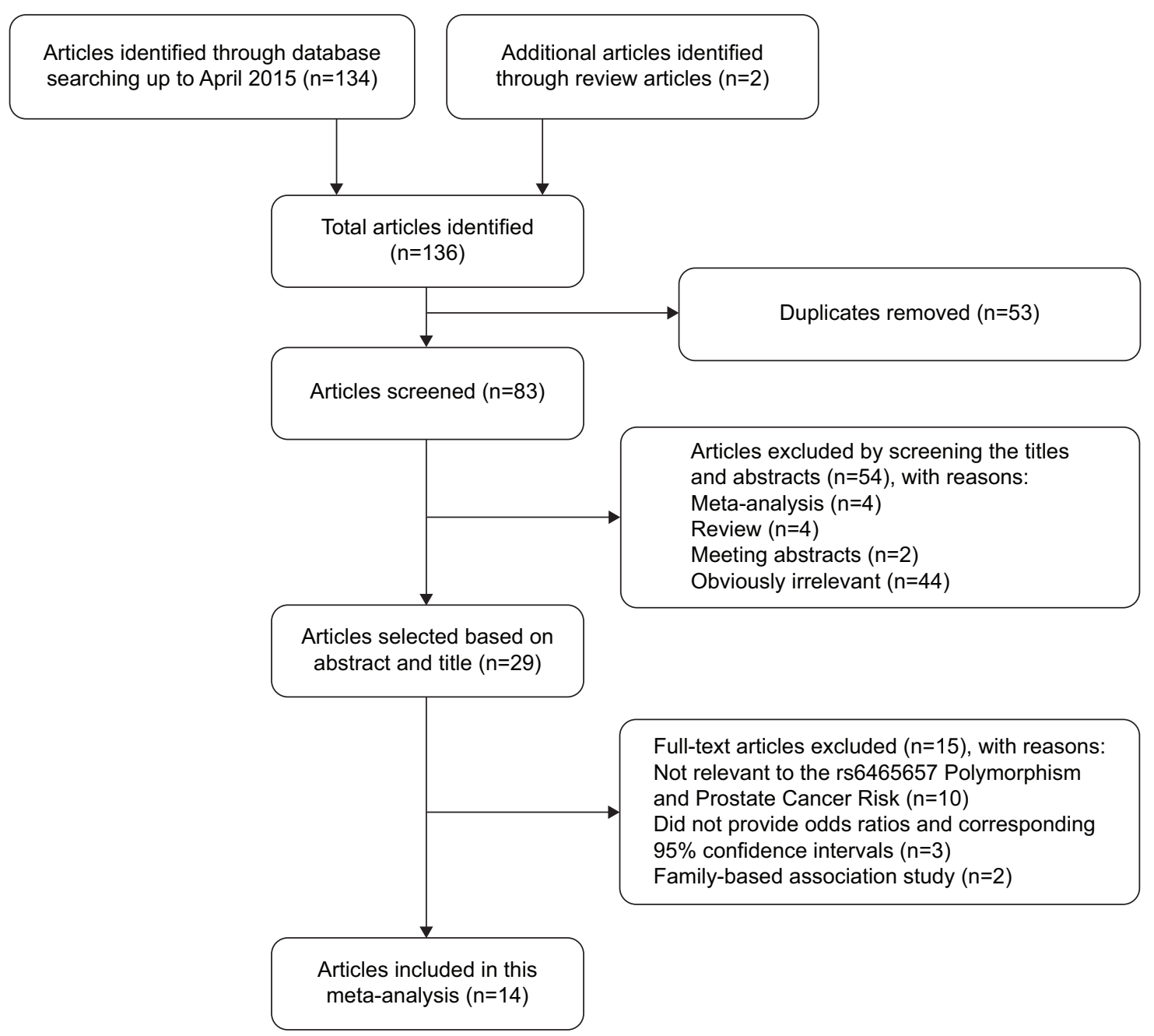

Figure I Flow diagram of meta-analysis for exclusion or inclusion of individual articles.

the overall and Caucasian populations under the allele model. Meanwhile, significant heterogeneity also came into play. The values of $Q$ were $33.33\left(I^{2}=46.0 ; P<0.05\right)$ in the overall population, $16.41\left(I^{2}=51.3 ; P<0.05\right)$ in Caucasians, $3.49\left(I^{2}=0 ; P>0.05\right)$ in Asians, and $3.25\left(I^{2}=69.3 ; P>0.05\right)$ in African-Americans, respectively. We performed a metaregression to determine the source of the heterogeneity in common variables. However, the year of publication, the ethnicity (Caucasian, Asian, or African-American), source of controls, genotyping method, and sample size (defined as sample size less than 500 cases, or more than 500 cases) were not correlated with heterogeneity $(P>0.05)$ based on statistical difference by using the random-effects model.

Sensitivity analysis was conducted to estimate the stability of the pooled ORs by omitting one study each time. The results confirmed that the significant association between the rs6465657 polymorphism and the PCa risk was stable in the overall (ORs ranged from 1.091 [95\% CI: 1.063-1.119] to 1.106 [95\% CI: 1.070-1.142]; Figure 4A) and Caucasian populations (ORs ranged from 1.103 [95\% CI: 1.079-1.130] to 1.124 [95\% CI: 1.079-1.170]; Figure 4B).

\section{Cumulative meta-analysis}

The cumulative meta-analysis revealed the trend of the association between rs6465657 polymorphism and PCa susceptibility under the allele model. With the sample size increasing, the pooled results reached statistical significance and the 95\% CIs narrowed down. It has been suggested that the results tended to be stabilized and reliable in both the overall (Figure S1A) and Caucasian populations (Figure S1B).

\section{Publication bias}

Begg's funnel plot and Egger's regression were conducted to evaluate the publication bias of the literature included in this meta-analysis. The shape of the Begg's test appeared symmetrical, indicating that no statistically significant evidence 


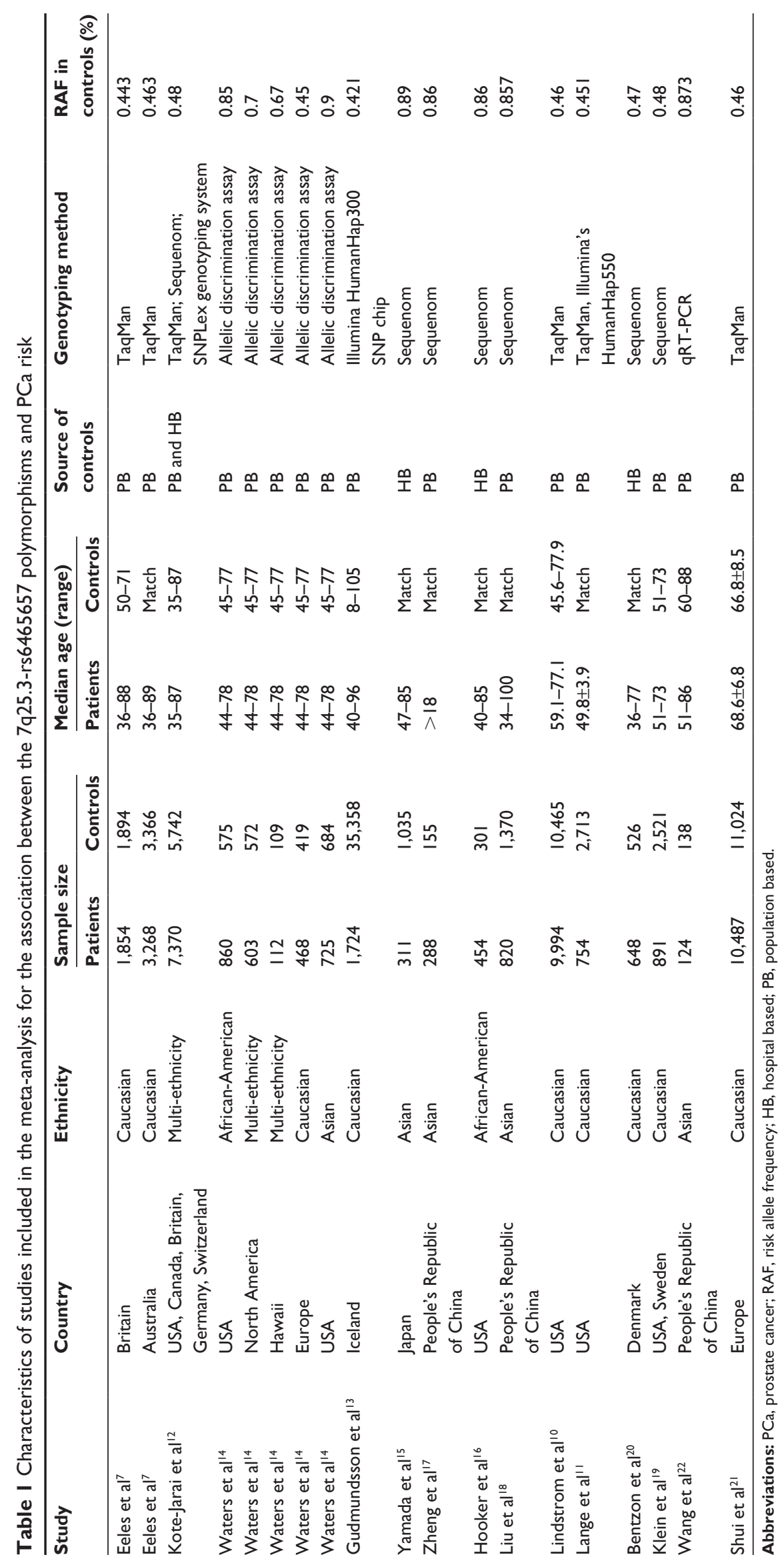




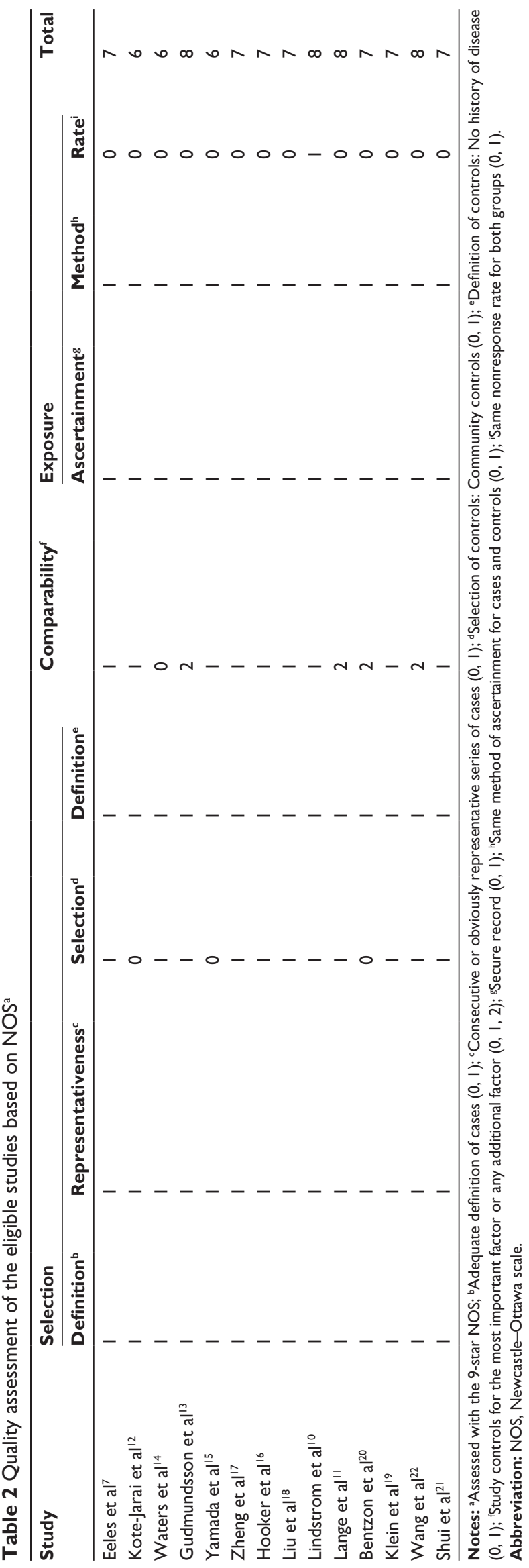

of publication bias was detected among the overall $(z=1.15$, $P>0.05$; Figure $\mathrm{S} 2 \mathrm{~A})$ or the Caucasian populations $(z=0.21$, $P>0.05$; Figure S2B). Egger's test provided consistent results in the overall $(t=-1.48, P>0.05$; Figure $\mathrm{S} 2 \mathrm{C})$ and the Caucasian populations ( $t=0.34, P>0.05$; Figure $\mathrm{S} 2 \mathrm{D})$.

\section{Discussion}

$\mathrm{PCa}$, the most commonly diagnosed cancer in males, is the second leading cause of cancer death among men in developed countries. ${ }^{2}$ Strong evidence from familial and epidemiologic studies supports the genetic predisposition to $\mathrm{PCa},{ }^{39}$ giving rise to the need for more studies to identify the full genetic profile of $\mathrm{PCa}$. However, genetic predisposition to $\mathrm{PCa}$ rarely corresponds to a high-penetrance Mendelian pattern of inheritance.

Recent GWAS studies identified multiple common genetic variants associated with PCa risk. To date, 77 susceptibility loci for PCa have been verified, and most of the loci were found in people of European descent. ${ }^{4}$ Among these loci, a SNP, rs6465657 was first demonstrated to be related to the increased risk of PCa in a GWAS study. ${ }^{7}$ Since then, the association between the 17q25.3-rs6465657 polymorphism and PCa susceptibility was evaluated independently by replication studies in Caucasians and other ethnicities, but consistent results have not been obtained.

Only one meta-analysis studying the association between the rs6465657 polymorphism and $\mathrm{PCa}$ risk has been published so far. ${ }^{23}$ It was based on the analysis of six articles ${ }^{7,10,13,14,16,17}$ involving 12 case-control studies. However, 14 articles providing 19 studies were obtained in the current meta-analysis, and the sample size increased from 66,901 to 120,722 . In addition, the relationship between rs6465657C and PCa risk did not reach statistical significance in the previous analysis due to the restriction of the sample size. Therefore, it was necessary for us to conduct an updated meta-analysis to reassess this association with more studies and subjects. In the present analysis, sensitivity analysis, Egger's test, and cumulative meta-analysis were also carried out to estimate the stability of the results. These aforementioned methods were not taken into consideration in the previous meta-analysis. ${ }^{23}$

In the analysis of the prevalence of $\mathrm{C}$ allele, the risk allele frequencies of controls varied wildly in different ethnicities; it was $45.6 \%$ in Caucasians, $87.9 \%$ in Asians, and $85.7 \%$ in African-Americans, respectively. This could be explained by the limited number of studies in Asians and African-Americans, which might not reflect the facticity about the prevalence rate of the risk allele. Furthermore, some important environmental factors such as lifestyle and 


\begin{tabular}{|c|c|c|c|c|}
\hline \multicolumn{3}{|l|}{ Study ID } & \multirow[t]{2}{*}{$\operatorname{RAF}(95 \% \mathrm{Cl})$} & Weight (\%) \\
\hline Caucasian & & & & \\
\hline Eeles et $\mathrm{al}^{7}$ & $\bullet$ & & $0.44(0.42-0.47)$ & 5.31 \\
\hline Eeles et $\mathrm{al}^{7}$ & $\bullet$ & & $0.46(0.45-0.48)$ & 5.32 \\
\hline Waters et $\mathrm{al}^{14}$ & $\rightarrow$ & & $0.45(0.40-0.50)$ & 5.21 \\
\hline Gudmundsson et $\mathrm{al}^{13}$ & $\bullet$ & & $0.42(0.42-0.43)$ & 5.33 \\
\hline Lindstrom et $\mathrm{al}^{10}$ & $\bullet$ & & $0.46(0.45-0.47)$ & 5.33 \\
\hline Lange et $\mathrm{al}^{11}$ & $\bullet$ & & $0.45(0.43-0.47)$ & 5.31 \\
\hline Bentzon et $\mathrm{al}^{20}$ & $\rightarrow$ & & $0.47(0.43-0.51)$ & 5.23 \\
\hline Klein et $\mathrm{al}^{19}$ & $\bullet$ & & $0.48(0.46-0.50)$ & 5.31 \\
\hline Shui et $a^{21}$ & $\bullet$ & & $0.46(0.45-0.47)$ & 5.33 \\
\hline Subtotal $\left(I^{2}=93.0 \%, P=0.000\right)$ & 0 & & $0.46(0.44-0.47)$ & 47.68 \\
\hline & & & & \\
\hline Asian & & & & \\
\hline Waters et al $^{14}$ & & $\bullet$ & $0.90(0.88-0.92)$ & 5.31 \\
\hline Yamada et $\mathrm{al}^{15}$ & & $\bullet$ & $0.89(0.87-0.91)$ & 5.31 \\
\hline Zheng et $\mathrm{al}^{17}$ & & $\rightarrow$ & $0.86(0.81-0.91)$ & 5.17 \\
\hline Liu et $\mathrm{al}^{18}$ & & $\bullet$ & $0.86(0.84-0.88)$ & 5.31 \\
\hline Wang et $\mathrm{al}^{22}$ & & 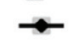 & $0.87(0.82-0.93)$ & 5.17 \\
\hline Subtotal $\left(I^{2}=62.1 \%, P=0.032\right)$ & & 0 & $0.88(0.86-0.90)$ & 26.27 \\
\hline & & & & \\
\hline Atrican-Americans & & & & \\
\hline Waters et al ${ }^{14}$ & & $\leftarrow$ & $0.85(0.82-0.88)$ & 5.29 \\
\hline Hooker et al ${ }^{16}$ & & $\rightarrow$ & $0.86(0.82-0.90)$ & 5.25 \\
\hline Subtotal $\left(I^{2}=0.0 \%, P=0.688\right)$ & & $\Delta$ & $0.85(0.83-0.88)$ & 10.54 \\
\hline & & & & \\
\hline Multi-ethnicity & & & & \\
\hline Kote-Jarai et al ${ }^{12}$ & $\bullet$ & & $0.48(0.47-0.49)$ & 5.32 \\
\hline Waters et $\mathrm{al}^{14}$ & & & $0.70(0.66-0.74)$ & 5.26 \\
\hline Waters et $\mathrm{al}^{14}$ & & & $0.67(0.58-0.76)$ & 4.93 \\
\hline Subtotal $\left(I^{2}=98.5 \%, P=0.000\right)$ & & & $0.61(0.44-0.79)$ & 15.51 \\
\hline Overall $\left(I^{2}=99.7 \%, P=0.000\right)$ & & & $0.63(0.56-0.70)$ & 100 \\
\hline
\end{tabular}

Figure 2 Frequencies of the C risk allele of 17q25.3-rs6465657 among controls stratified by ethnicity.

Note: Weights are from random-effects analysis.

Abbreviations: $\mathrm{Cl}$, confidence interval; $\mathrm{RAF}$, risk allele frequency.

diet might have an impact on the risk allele prevalence among different populations.

The current meta-analysis is the most comprehensive one evaluating the association between 17q25.3-rs6465657 polymorphism and $\mathrm{PCa}$ susceptibility. The overall analysis suggested that the $\mathrm{C}$ allele of rs6465657 significantly associated with the increased risk of PCa. In the subgroup analysis stratified by ethnicity, the rs6465657C showed a positive association with PCa risk in Caucasians, whereas the association in Asian and African-American populations did not reach statistical significance. Some possible reasons may be responsible for the results. First, due to the limited sample size in people of Asian and African-American descents, the eligible studies did not have sufficient statistical power to detect a slight effect of the risk allele on the susceptibility of $\mathrm{PCa}$. Second, if the $\mathrm{C}$ allele is an extremely common genetic variant in Asians and Africans, it is implied that the variation in this locus might not be a risk factor, but a universal SNP that exists in these two ethnicities. Third, both the genetic background and the environmental factors may contribute to the different effects of the risk allele on the occurrence of $\mathrm{PCa}$ among different populations, which may explain the opposite results observed between Caucasians and other populations to some extent. Given these potential reasons described, we thought that the rs6465657C was probably associated with occurrence of $\mathrm{PCa}$, especially in Caucasians, while this association varies in other ethnic populations.

Cumulative analysis showed that the 95\% CI narrowed down with the increase of the sample size, which demonstrated that the effect of rs6465657C allele on PCa risk was more potent when the number of study subjects was increased in this meta-analysis. In addition, sensitivity analysis and publication bias analysis indicated the robust stability of our results.

The rs6465657 polymorphism at chromosome $7 \mathrm{q} 21.3$ is found in 9 intron of LMTK2. The protein encoded by LMTK2 


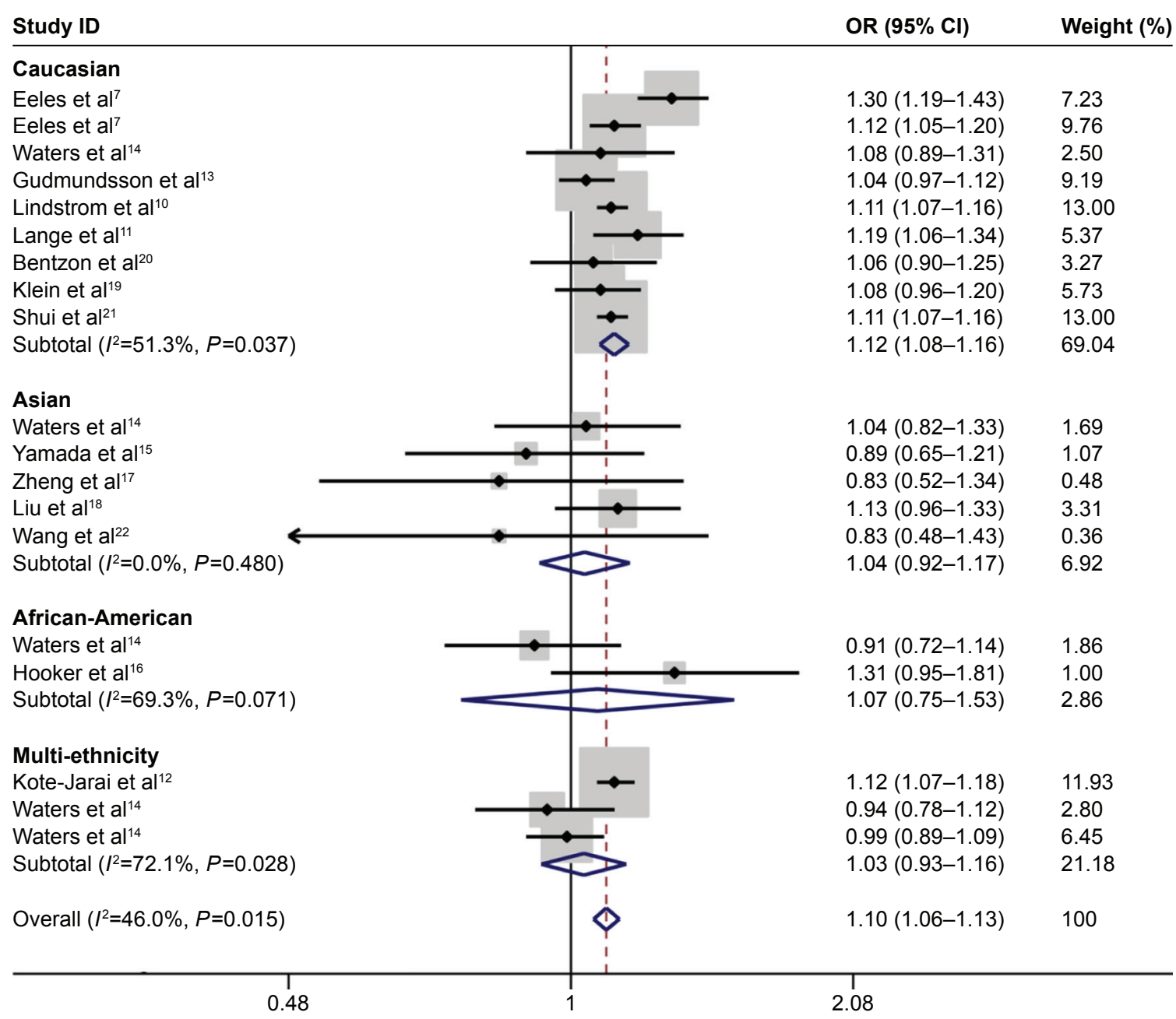

Figure 3 Forest plot for association of 17q25.3-rs6465657 polymorphism and PCa risk.

Note: Weights are from random-effects analysis.

Abbreviations: $\mathrm{OR}$, odds ratio; $\mathrm{Cl}$, confidence interval; $\mathrm{PCa}$, prostate cancer.

gene is a tyrosine kinase belonging to the lemur membrane associated kinase family ${ }^{40}$ and is reported to be involved in PCa. ${ }^{41}$ Recently, a study conducted by Harries et $\mathrm{al}^{9}$ suggested a $68 \%$ reduction in the expression of the LMTK2 gene in PCa tissue in contrast to nonmalignant samples. Puri et $\mathrm{al}^{42}$ have revealed that LMTK2 may interact with myosin IV, and this protein has been identified to regulate prostate specific antigen (PSA) and vascular endothelial growth factor (VEGF) which are related to tumorigenesis. It has also been demonstrated that loss of LMTK2 leads to an increase in cell proliferation and tumor-forming capacity in prostate adenocarcinoma cell lines (LNCaP cells). ${ }^{43}$ Given that LMTK2 gene has substantial influence on PCa and that the rs6465657 polymorphism in intron 9 of LMTK2 has been identified, the association with PCa risk in a GWAS study, the precise mechanism of the underlying relationship between LMTK2 and rs6465657 polymorphism should be further evaluated. It is suggested that the rs6465657C allele might contribute

Table 3 Results of meta-analysis for 17q25.3-rs6465657 polymorphism with PCa risk

\begin{tabular}{|c|c|c|c|c|c|c|c|c|c|c|}
\hline \multirow{2}{*}{$\begin{array}{l}\text { Total and } \\
\text { subgroups }\end{array}$} & \multirow{2}{*}{$\begin{array}{l}\text { Number } \\
\text { of studies }\end{array}$} & \multicolumn{2}{|c|}{ Sample sizes } & \multicolumn{3}{|c|}{ Test of heterogeneity } & \multicolumn{4}{|c|}{ Test of association } \\
\hline & & Patients & Controls & $\bar{Q}$ & $P$-value & $\overline{I^{2}(\%)}$ & $\overline{\text { OR }}$ & $95 \% \mathrm{Cl}$ & $Z$ & $P$-value \\
\hline Total & 19 & 41,755 & 78,967 & 33.33 & 0.02 & 46.0 & 1.097 & $1.061-1.134$ & 5.44 & 0.000 \\
\hline \multicolumn{11}{|l|}{ Ethnicity } \\
\hline Caucasian & 9 & 30,088 & 68,286 & $16.4 \mid$ & 0.04 & 51.3 & 1.120 & $1.078-1.162$ & 5.91 & 0.000 \\
\hline Asian & 5 & 2,268 & 3,382 & 3.49 & 0.48 & 0.0 & 1.036 & $0.922-1.165$ & 0.6 & 0.550 \\
\hline African-American & 2 & $|, 3| 4$ & 876 & 3.25 & 0.07 & 69.3 & 1.072 & $0.752-1.529$ & 0.38 & 0.701 \\
\hline
\end{tabular}

Note: A $P$-value $<0.05$ was considered to be statistically significant.

Abbreviations: PCa, prostate cancer; OR, odds ratio; $\mathrm{Cl}$, confidence interval. 
A

Meta-analysis estimates, given named study is omitted

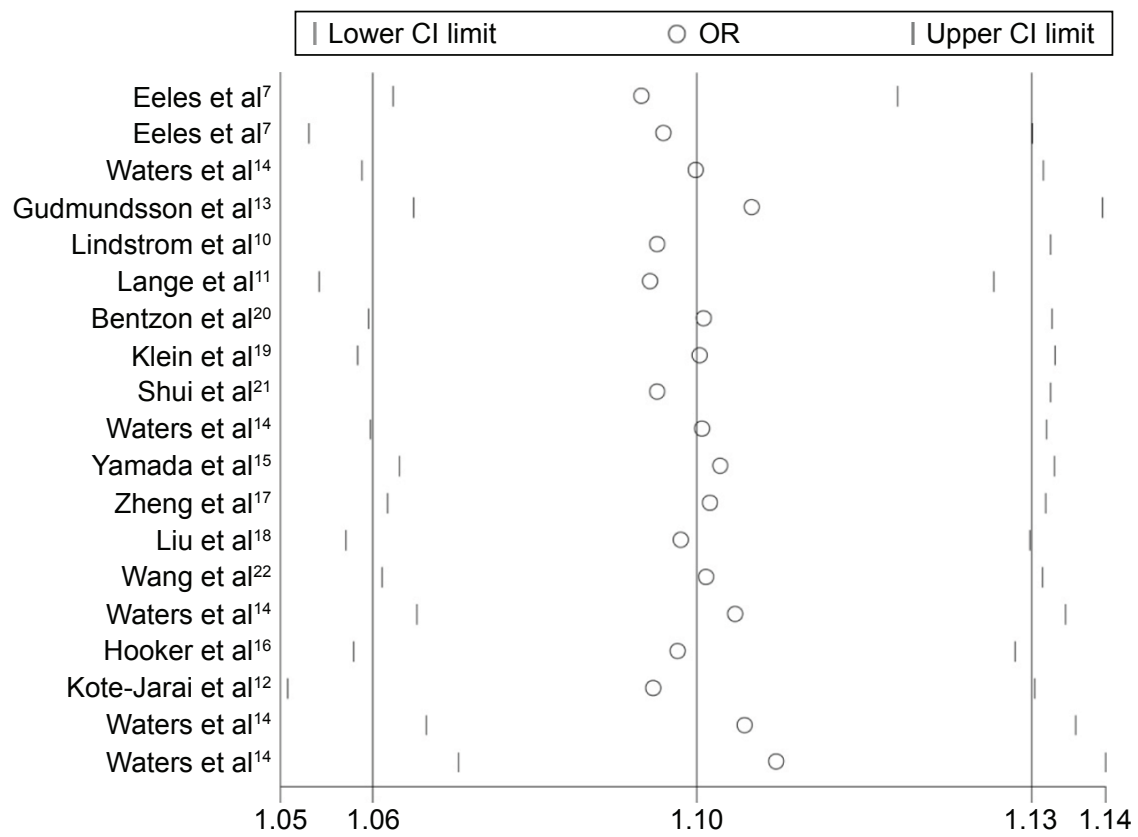

B

Meta-analysis estimates, given named study is omitted

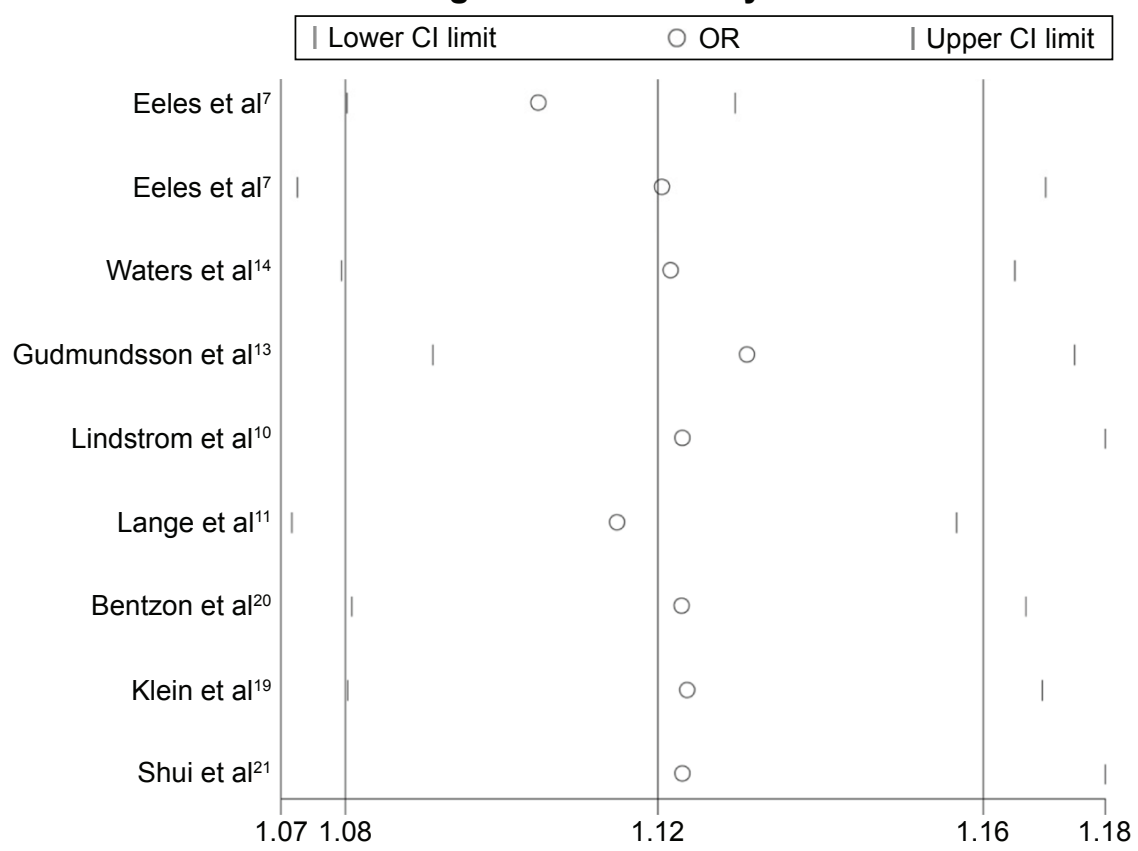

Figure 4 Sensitivity analysis in the overall population (A) and Caucasian population (B) using the random-effects model.

Notes: This was done to evaluate the influence of each study on the stability of pooled OR by removing one study in turn and recalculating the ORs and $95 \%$ Cls. The vertical axis in the middle of the figure represents the pooled OR and the two vertical axes located on either side represent the corresponding $95 \% \mathrm{Cl}$. Meanwhile, the closed circles are representative of the values of pooled ORs with the study mentioned on the left side of the figure being omitted. The two ends of each dotted line represent its corresponding $95 \% \mathrm{Cl}$.

Abbreviations: $\mathrm{Cl}$, confidence interval; OR, odds ratio.

to a loss of function which may affect LMTK2 half-life or other functions. ${ }^{9}$ Further studies are needed to investigate the possible mechanism by which rs 6465657 polymorphism regulates the $L M T K 2$ gene and the mechanism by which LMTK2 contributes to PCa development.

\section{Limitations}

Some limitations of this meta-analysis should also be acknowledged. First, significant heterogeneity was detected in the overall and the subgroup analysis. Although meta-regression was conducted to look into the source of 
heterogeneity in common variables, the year of publication, ethnicity, sample size, genotyping method, and source of controls were not correlated to heterogeneity $(P>0.05)$. Second, the recessive, dominant, heterozygous, and homozygous models were not mentioned in our meta-analysis due to the lack of original data in GWAS and replication studies. Third, the inherent confounding factors in the included studies could not be solved by meta-analysis. Although the evaluations from all the eligible studies were adjusted for some possible risk factors leading to PCa susceptibility, there might be other unknown confounders in the controls that could not be excluded, which might contribute to the bias observed in the results. Finally, the background frequency of rs6465657C varies wildly among different populations. In particular, the suspected risk allele was extremely common in African-American and Asian populations. This meant that there would be insufficient statistical power to find a small effect of rs6465657C on PCa risk with the high prevalence rate of risk allele in Asian or African-American controls and that the rs6465657C allele might not be the risk factor to $\mathrm{PCa}$ in Asians and African-Americans.

\section{Conclusion}

Our meta-analysis provided a comprehensive evaluation of the association between 17q25.3-rs6465657 polymorphism and PCa risk. Our results suggested that rs6465657C allele contributed to the susceptibility to $\mathrm{PCa}$, especially in Caucasians. Well-designed studies with larger sample size and more ethnic groups are required to validate the risk in the future, and biological mechanisms contributing to this association also need to be focused on.

\section{Acknowledgments}

This research was supported by grants from the National Nature Science Foundation of China (31271500 to H.W., and 31301159 to S.L.) and from Liaoning Innovative Research Team in University (LT2015008 to H.W.).

\section{Disclosure}

The authors report no conflicts of interests in this work.

\section{References}

1. Heidenreich A, Bastian PJ, Bellmunt J, et al. EAU guidelines on prostate cancer. Part 1: screening, diagnosis, and local treatment with curative intent-update 2013. Euro Urol. 2014;65(1):124-137.

2. Torre LA, Bray F, Siegel RL, Ferlay J, Lortet-Tieulent J, Jemal A. Global cancer statistics, 2012. CA Cancer J Clin. 2015;65(2):87-108.

3. Kim S, Shin C, Jee SH. Genetic variants at 1q32. 1, 10q11. 2 and 19q13. 41 are associated with prostate-specific antigen for prostate cancer screening in two Korean population-based cohort studies. Gene. 2015;556(2): 199-205.
4. Eeles RA, Al Olama AA, Benlloch S, et al. Identification of 23 new prostate cancer susceptibility loci using the iCOGS custom genotyping array. Nat Genet. 2013;45(4):385-391.

5. Lichtenstein P, Holm NV, Verkasalo PK, et al. Environmental and heritable factors in the causation of cancer - analyses of cohorts of twins from Sweden, Denmark, and Finland. N Engl J Med. 2000; 343(2):78-85.

6. Hjelmborg JB, Scheike T, Holst K, et al. The heritability of prostate cancer in the Nordic Twin Study of Cancer. Cancer Epidemiol Biomarkers Prev. 2014;23(11):2303-2310.

7. Eeles RA, Kote-Jarai Z, Giles GG, et al. Multiple newly identified loci associated with prostate cancer susceptibility. Nat Genet. 2008;40(3): 316-321.

8. Eeles RA, Kote-Jarai Z, Al Olama AA, et al. Identification of seven new prostate cancer susceptibility loci through a genome-wide association study. Nat Genet. 2009;41(10):1116-1121.

9. Harries LW, Perry JR, McCullagh P, Crundwell M. Alterations in LMTK2, MSMB and HNF1B gene expression are associated with the development of prostate cancer. BMC Cancer. 2010;10(1):315.

10. Lindstrom S, Schumacher F, Siddiq A, et al. Characterizing associations and SNP-environment interactions for GWAS-identified prostate cancer risk markers - results from BPC3. PLoS One. 2011;6(2):e17142.

11. Lange EM, Salinas CA, Zuhlke KA, et al. Early onset prostate cancer has a significant genetic component. Prostate. 2012;72(2):147-156.

12. Kote-Jarai Z, Easton DF, Stanford JL, et al. Multiple novel prostate cancer predisposition loci confirmed by an international study: the PRACTICAL Consortium. Cancer Epidemiol Biomarkers Prev. 2008; 17(8):2052-2061.

13. Gudmundsson J, Sulem P, Gudbjartsson DF, et al. Genome-wide association and replication studies identify four variants associated with prostate cancer susceptibility. Nat Genet. 2009;41(10):1122-1126.

14. Waters KM, Le Marchand L, Kolonel LN, et al. Generalizability of associations from prostate cancer genome-wide association studies in multiple populations. Cancer Epidemiol Biomarkers Prev. 2009;18(4): 1285-1289.

15. Yamada H, Penney KL, Takahashi H, et al. Replication of prostate cancer risk loci in a Japanese case-control association study. $J$ Natl Cancer Inst. 2009;101(19):1330-1336.

16. Hooker S, Hernandez W, Chen H, et al. Replication of prostate cancer risk loci on 8q24, 11q13, 17q12, 19q33, and Xp11 in African Americans. Prostate. 2010;70(3):270-275.

17. Zheng SL, Hsing AW, Sun J, et al. Association of 17 prostate cancer susceptibility loci with prostate cancer risk in Chinese men. Prostate. 2010;70(4):425-432.

18. Liu F, Hsing AW, Wang X, et al. Systematic confirmation study of reported prostate cancer risk-associated single nucleotide polymorphisms in Chinese men. Cancer Sci. 2011;102(10):1916-1920.

19. Klein RJ, Hallden C, Gupta A, et al. Evaluation of multiple riskassociated single nucleotide polymorphisms versus prostate-specific antigen at baseline to predict prostate cancer in unscreened men. Eur Urol. 2012;61(3):471-477.

20. Bentzon DN, Nyegaard M, Børglum A, Ørntoft T, Borre M, Sørensen KD. Replication of prostate cancer risk variants in a Danish case-control association study. Open J Urol. 2012;02(02):45-54.

21. Shui IM, Lindstrom $\mathrm{S}$, Kibel AS, et al. Prostate cancer (PCa) risk variants and risk of fatal PCa in the National Cancer Institute Breast and Prostate Cancer Cohort Consortium. Eur Urol. 2014;65(6): 1069-1075.

22. Wang N, Wang J, Shi X, et al. [Association of TET2, LMTK2 and FAM84B gene expression with prostate cancer risk in Chinese patients]. Zhonghua Zhong Liu Za Zhi. 2013;35(4):262-267. Chinese.

23. Liu H, Wang B, Han C. Meta-analysis of genome-wide and replication association studies on prostate cancer. Prostate. 2011;71(2): 209-224.

24. Stang A. Critical evaluation of the Newcastle-Ottawa scale for the assessment of the quality of nonrandomized studies in meta-analyses. Eur J Epidemiol. 2010;25(9):603-605. 
25. Bai XY, Li S, Wang M, et al. Association of monocyte chemoattractant protein-1 (MCP-1)-2518A $>$ G polymorphism with susceptibility to coronary artery disease: a meta-analysis. Ann Hum Genet. 2015;79(3): 173-187.

26. Bowden J, Tierney JF, Copas AJ, Burdett S. Quantifying, displaying and accounting for heterogeneity in the meta-analysis of RCTs using standard and generalised Q statistics. BMC Med Res Methodol. 2011;11:41.

27. Mantel N. Chi-square tests with one degree of freedom; extensions of the Mantel-Haenszel procedure. J Am Stat Assoc. 1963;58(303):690-700.

28. DerSimonian R, Laird N. Meta-analysis in clinical trials. Control Clin Trials. 1986;7(3):177-188.

29. Zabalza M, Subirana I, Sala J, et al. Meta-analyses of the association between cytochrome CYP2C19 loss-and gain-of-function polymorphisms and cardiovascular outcomes in patients with coronary artery disease treated with clopidogrel. Heart. 2012;98(2):100-108.

30. Cochran WG. The combination of estimates from different experiments. Biometrics. 1954;10(1):101-129.

31. Begg CB, Mazumdar M. Operating characteristics of a rank correlation test for publication bias. Biometrics. 1994;50(4):1088-1101.

32. Egger M, Smith GD, Schneider M, Minder C. Bias in meta-analysis detected by a simple, graphical test. BMJ. 1997;315(7109):629-634.

33. Pomerantz MM, Freedman ML. Genetics of prostate cancer risk. Mt Sinai J Med. 2010;77(6):643-654.

34. Takata R, Akamatsu S, Kubo M, et al. Genome-wide association study identifies five new susceptibility loci for prostate cancer in the Japanese population. Nat Genet. 2010;42(9):751-754.

35. Bao BY, Pao JB, Lin VC, et al. Individual and cumulative association of prostate cancer susceptibility variants with clinicopathologic characteristics of the disease. Clin Chim Acta. 2010;411(17-18):1232-1237.
36. Rinckleb AE. Common Germline Variants for Prostate Cancer Risk: Implication in DNA Repair and TMPRSS2-ERG Fusion Formation [PhD dissertation]. Ulm, Germany: Faculty of Medicine, Ulm University; 2014.

37. Macinnis RJ, Antoniou AC, Eeles RA, et al. A risk prediction algorithm based on family history and common genetic variants: application to prostate cancer with potential clinical impact. Genet Epidemiol. 2011; 35(6):549-556.

38. Camp NJ, Farnham JM, Wong J, Christensen GB, Thomas A, CannonAlbright LA. Replication of the $10 \mathrm{q} 11$ and Xp11 prostate cancer risk variants: results from a Utah pedigree-based study. Cancer Epidemiol Biomarkers Prev. 2009;18(4):1290-1294.

39. Edwards SM, Kote-Jarai Z, Meitz J, et al. Two percent of men with early-onset prostate cancer harbor germline mutations in the BRCA2 gene. Am J Hum Genet. 2003;72(1):1-12.

40. Wang H, Brautigan DL. A novel transmembrane Ser/Thr kinase complexes with protein phosphatase-1 and inhibitor-2. J Biol Chem. 2002;277(51):49605-49612.

41. Nixon A, Jia Y, White C, Bradbury NA. Determination of the membrane topology of lemur tyrosine kinase 2 (LMTK2) by fluorescence protease protection. Am J Physiol Cell Physiol. 2013;304(2):C164-C169.

42. Puri C, Chibalina MV, Arden SD, Kruppa AJ, Kendrick-Jones J, Buss F. Overexpression of myosin VI in prostate cancer cells enhances PSA and VEGF secretion, but has no effect on endocytosis. Oncogene. 2010;29(2):188-200.

43. Shah K, Bradbury N. LMTK2, a novel target in prostate cancer therapy. FASEB J. 2015;29(1 Suppl):974.910. 


\section{Supplementary materials}

A Study ID

Waters et $\mathrm{al}^{14}$

Wang et $\mathrm{al}^{22}$

Zheng et al ${ }^{17}$

Hooker et al ${ }^{16}$

Waters et $\mathrm{al}^{14}$

Bentzon et $\mathrm{al}^{20}$

Waters et al ${ }^{14}$

Yamada et $\mathrm{al}^{15}$

Waters et $\mathrm{al}^{14}$

Waters et $\mathrm{al}^{14}$

Liu et al ${ }^{18}$

Klein et al ${ }^{19}$

Lange et $\mathrm{al}^{11}$

Eeles et $\mathrm{al}^{7}$

Eeles et $\mathrm{al}^{7}$

Kote-Jarai et $\mathrm{al}^{12}$

Lindstrom et $\mathrm{al}^{10}$

Shui et $\mathrm{al}^{21}$

Gudmundsson et al ${ }^{13}$
OR $(95 \% \mathrm{Cl})$

$0.99(0.89-1.10)$

$0.98(0.89-1.09)$

$0.98(0.89-1.08)$

$1.01(0.87-1.18)$

$1.02(0.93-1.12)$

$1.02(0.95-1.10)$

$1.01(0.94-1.08)$

$1.01(0.94-1.08)$

$1.01(0.94-1.08)$

$1.00(0.94-1.07)$

$1.02(0.96-1.08)$

$1.03(0.98-1.08)$

$1.05(1.00-1.11)$

$1.07(1.00-1.15)$

$1.08(1.02-1.15)$

$1.09(1.04-1.15)$

$1.10(1.05-1.14)$

$1.10(1.07-1.14)$

$1.10(1.06-1.13)$

B

Study ID

OR $(95 \% \mathrm{Cl})$

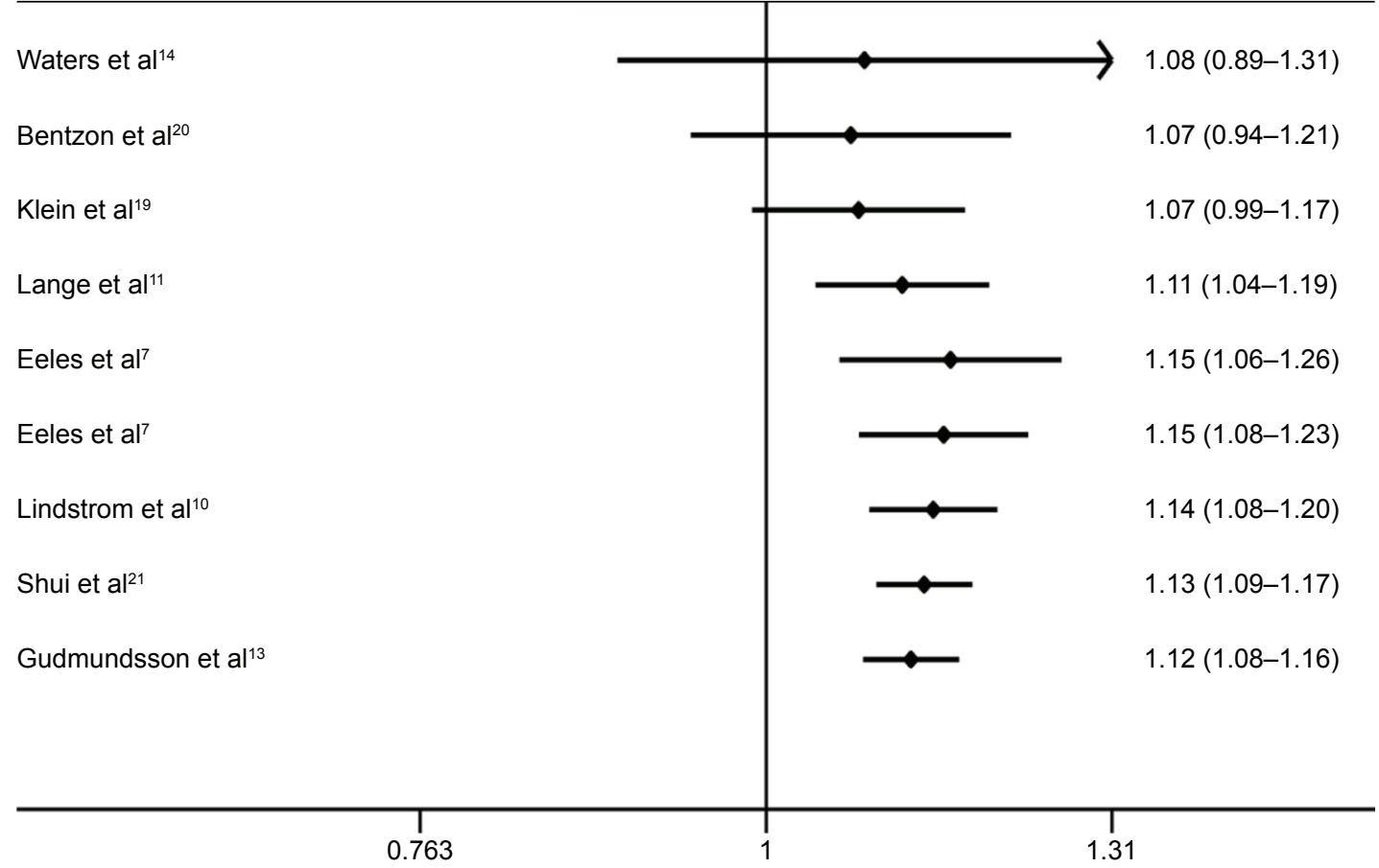

Figure SI Cumulative meta-analysis of the association between I7q25.3-rs6465657C and susceptibility to PCa in the overall (A) and Caucasian population (B) sorted by sample size.

Abbreviations: PCa, Prostate cancer; OR, odds ratio; Cl, confidence interval. 

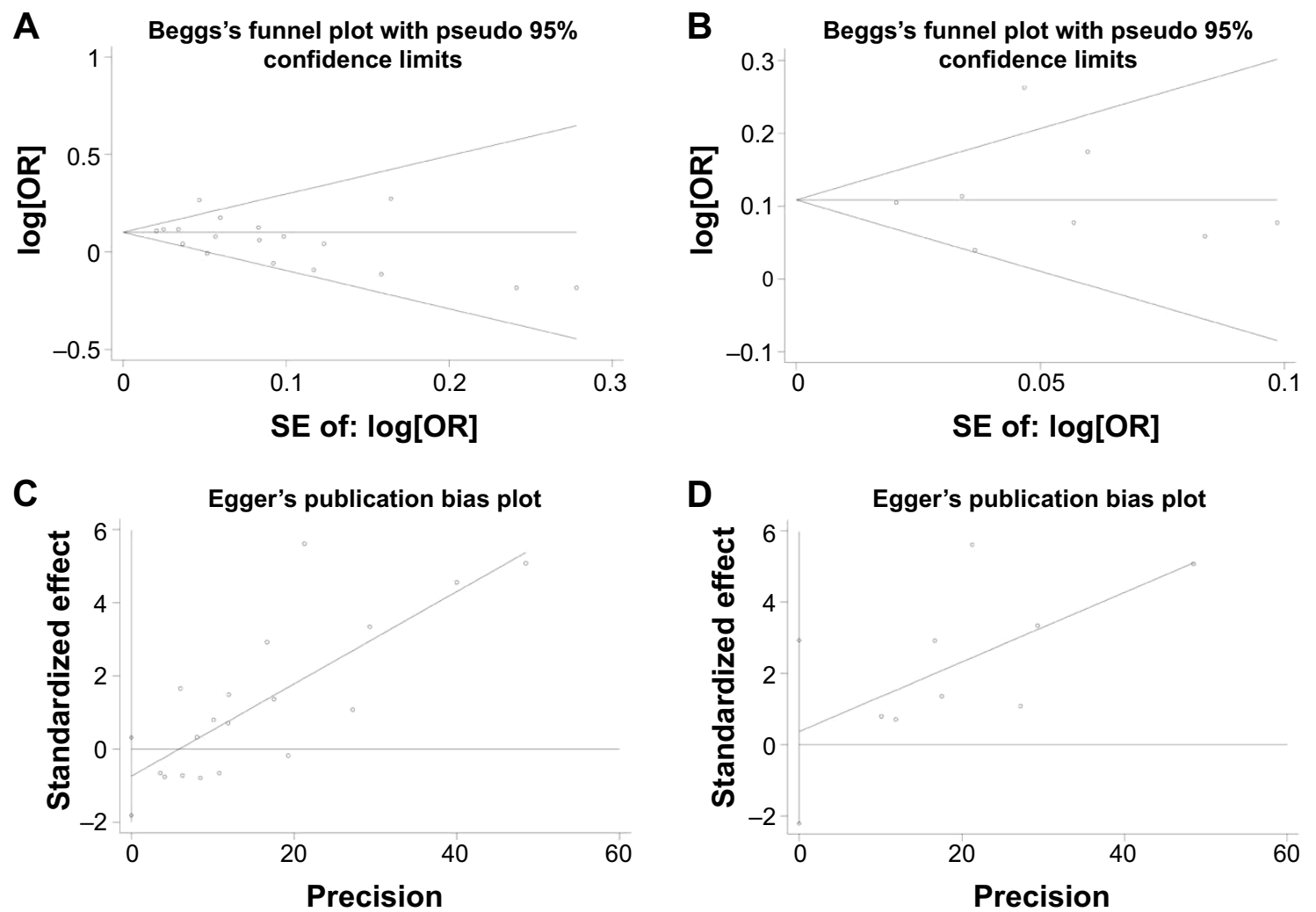

Figure S2 Begg's funnel plot applied for evaluating publication bias in the overall population and Caucasian population (A, B). Egger's regression was also performed (C, D). Abbreviations: OR, odds ratio; SE, standard error.

\section{Publish your work in this journal}

OncoTargets and Therapy is an international, peer-reviewed, open access journal focusing on the pathological basis of all cancers, potential targets for therapy and treatment protocols employed to improve the management of cancer patients. The journal also focuses on the impact of management programs and new therapeutic agents and protocols on patient perspectives such as quality of life, adherence and satisfaction. The manuscript management system is completely online and includes a very quick and fair peer-review system, which is all easy to use. Visit http://www.dovepress.com/testimonials.php to read real quotes from published authors. 